\title{
Variable Survival Exponents in History-Dependent Random Walks: Hard Movable Reflector
}

\author{
Ronald Dickman ${ }^{1}$, Francisco Fontenele Araujo Jr. ${ }^{1}$, and Daniel ben-Avraham ${ }^{2}$ \\ ${ }^{1}$ Departamento de Física, ICEx, Universidade Federal de Minas Gerais, \\ C.P. 702, 30.161-970, Belo Horizonte - Minas Gerais, Brazil \\ ${ }^{2}$ Physics Department, Clarkson University, Potsdam, NY 13699-5820, USA
}

Received on 1st April, 2003

\begin{abstract}
We review recent studies demonstrating a nonuniversal (continuously variable) survival exponent for historydependent random walks, and analyze a new example, the hard movable partial reflector. These processes serve as simplified models of infection in a medium with a history-dependent susceptibility, and for spreading in systems with an infinite number of absorbing configurations. The memory may take the form of a historydependent step length, or be the result of a partial reflector whose position marks the maximum distance the walker has ventured from the origin. In each case, a process with memory is rendered Markovian by a suitable expansion of the state space. Asymptotic analysis of the probability generating function shows that, for large $t$, the survival probability decays as $S(t) \sim t^{-\delta}$, where $\delta$ varies with the parameters of the model. We report new results for a hard partial reflector, i.e., one that moves forward only when the walker does. When the walker tries to jump to the site $\mathrm{R}$ occupied by the reflector, it is reflected back with probability $r$, and stays at $\mathrm{R}$ with probability $1-r$; only in the latter case does the reflector move $(\mathrm{R} \rightarrow \mathrm{R}+1)$. For this model, $\delta=1 / 2(1-r)$, and becomes arbitrarily large as $r$ approaches 1 . This prediction is confirmed via iteration of the transition matrix, which also reveals slowly-decaying corrections to scaling.
\end{abstract}

\section{Introduction}

Random walks with absorbing or reflecting boundaries, or with memory, serve as important models in statistical physics, often admitting an exact analysis. Among the many examples are equilibrium models for polymer adsorption [14] and absorbing-state phase transitions [5]. Another motivation for the study of such problems is provided by the spreading of an epidemic in a medium with a long memory $[6,7]$.

In addition to the intrinsic interest of such an infection with memory, our study is motivated by the spread of activity in models exhibiting an infinite number of absorbing configurations (INAC), typified by the pair contact process $[8,9]$. Anomalies in critical spreading for INAC, such as continuously variable critical exponents, have been traced to a long memory in the dynamics of the order parameter, $\rho$, due to coupling to an auxiliary field that remains frozen in regions where $\rho=0[9,10]$. INAC appears to be particularly relevant to the transition to spatiotemporal chaos, as shown in a recent study of a coupled-map lattice with "laminar" and "turbulent" states, which revealed continuously variable spreading exponents [11].

Grassberger, Chaté and Rousseau [6] proposed that spreading in INAC could be understood by studying a model with a unique absorbing configuration, but in which the spreading rate of activity into previously inactive regions is different than for revisiting a region that has already been active. In light of the anomalies found in spreading in models with INAC or with a memory, we are interested in studying the effect of such a memory on the scaling behavior in a model whose asymptotic behavior can be determined exactly. Of particular interest is the survival probability $S(t)$ (i.e., not to have fallen into the absorbing state up to time $t$ ).

In the present work, we review previous results on survival of random walks with memory, and analyze the asymptotic behavior of a random walk subject to a hard movable reflector. On the basis of an exact solution for the probability generating function, we obtain the decay exponent $\delta$.

The balance of this paper is organized as follows. Section II reviews previous results on variable survival exponents for random walks with memory. In Sec. III we define the hard reflector model and obtain a formal solution for the generating function. An asymptotic analysis of this function is presented in Sec. IV, leading to an expression for the decay exponent $\delta$ in terms of the reflection probability $r$. In Sec. $\mathrm{V}$ we present exact numerical results for finite times (from iteration of the probability transfer matrix) that complement and extend the asymptotic analysis. Sec. VI contains a brief summary and discussion. 


\section{Variable survival exponents in ran- dom walks with memory}

Relatively simple random walk problems often serve as reduced examples of much more complicated many-body phemomena. So it is with phase transitions between an active and an absorbing state. In these systems $[5,12]$ the stationary state of a Markov process exhibits (in the infinite-size limit) a phase transition from a frozen, inactive regime to one with sustained activity, as a control parameter is varied. Broadly speaking, the control parameter represents the reproduction rate of activity $(\mathrm{A} \rightarrow 2 \mathrm{~A}$ ) relative to its extinction $(\mathrm{A} \rightarrow 0)$. The simplest examples are the contact process [13] and directed percolation (DP).

Consider now, instead of the stationary state, the spread of activity from a localized source, in an infinite system. In the subcritical regime (for which the only stationary state is the inactive, absorbing one), the initial activity decays (typically, exponentially fast), while in the supercritical regime there is a finite probability for it to spread indefinitely. Just at the critical point, one finds a scale-invariant evolution: the survival probability $S(t)$, the integrated activity $n(t)$, and the mean-square distance, $R^{2}(t)$, of the activity from the initial seed, all follow asymptotic power laws.

The question of survival arises naturally in the context of a random walk in the presence of an absorbing boundary. The survival probability $S(t)$ is the probability never to have visited an absorbing boundary until time $t$. The simplest example is a one-dimensional random walk $x_{t}$ (in discrete time) on the non-negative integers, with the origin absorbing, and $x_{0} \geq 1$. Let the walker jump, at each time step, to the right $\left(x_{t+1}=x_{t}+1\right)$ with probability $p$, and the left $\left(x_{t+1}=x_{t}-1\right)$ with probability $q=1-p$. If $p<q$ then the survival probability decays exponentially, while for $p>q$ it approaches (again exponentially) a nonzero value, so that the walker has a finite probability to escape to infinity. In the absence of drift ( $p=q=1 / 2), S(t) \sim t^{-1 / 2}$ for large $t$; associated with this power-law decay is an infinite mean lifetime. In the analogy with an absorbing-state phase transition, $p=1 / 2$ evidently marks the transition, with extinction certain for $p \leq 1 / 2$, and a finite asymptotic survival probability for $p>1 / 2$. The same qualitative situation holds in the contact process, starting for example from a single active site [5].

The analogy is in fact exact for the rather special case of compact directed percolation (CDP), in which active regions are delimited by independent random walks that annihilate on contact. (CDP is a particular limit of the DomanyKinzel model [14].) In this case 'drift' corresponds to a tendency of the walkers at the boundaries of an active region to approach, or separate from, one another; the critical point corresponds to zero drift, or unbiased random walkers $(p=1 / 2)$. Initializing CDP with a finite interval (say, $1,2, \ldots, \mathrm{m})$ of active sites, is equivalent to placing random walkers $\left(x_{t}\right.$ and $\left.y_{t}\right)$ at 0 and $m+1$. The active region at any subsequent time corresponds to the interval between the walkers $x_{t}$ and $y_{t}$; the process ends when the two meet. To make the analogy between CDP and a random walk with the origin absorbing complete, we may fix $x_{t}=0$ for all times, so that only the right frontier of the active region is free to fluctuate, while the left frontier is pinned, as it were, at a wall.

Given the connection with phase transitions, we shall think of the power law for the survival probabilty as defining a critical exponent, and write $S(t) \sim t^{-\delta}$. The main interest of the examples discussed in this paper is that the exponent $\delta$ can be shown to vary continuously as a function of a parameter. This in turn may help to understand scaling in more complex examples, such as the pair contact process [8], for which a variable survival exponent has been reported numerically, but cannot be established rigorously.

Random walk models exhibiting variable survival exponents fall in two classes. In one, the position of the absorbing boundary is a given (deterministic) function of time. A random walk in the presence of such a boundary defines a nonstationary stochastic process. The second class (which is our principal interest here) involves memory, either in the form of a reflector that moves when it encounters the walker, or of a history-dependent step length.

We begin with a brief review of the first class. In a highly readable paper, Krapivsky and Redner [15] considered what happens when a random walker on the line is subject to two absorbing boundaries, $R(t)$ and $L(t)$, which are prescribed functions of time (initially the walker is at the origin). The absorbers are initially near the origin, and follow $R(t)=a+(A t)^{\alpha}, L(t)=-R(t)$. For $\alpha>1 / 2$, the absorbers rapidly leave the region explored by the walker, which therefore enjoys a finite probability of survival as $t \rightarrow \infty$. If the absorbers are stationary we of course expect $S(t)$ to decay exponentially; but if their motion is characterized by $0<\alpha<1 / 2$, this changes to a stretched exponential decay, $S \sim \exp \left[-t^{1-2 \alpha}\right]$. For $\alpha=1 / 2$, the 'safe' region expands with the same power law as the region explored by the walker. The result is power-law decay of the survival probability, with a variable exponent which depends on $A$.

The problem studied by Krapivsky and Redner may be pictured as a random walk confined to a parabola in the $t-x$ plane, whose equation (for $t \geq 0$ ) is $x= \pm(A t)^{\alpha}$. When $\alpha=1 / 2$, as noted, there is power-law decay of $S(t)$, with a nonuniversal exponent. Similar conclusions apply for DP and for directed self-avoiding walks [16], and for CDP [17], when these processes are confined to a fixed parabola.

We now turn to studies of a random walk subject to some special condition when it enters virgin territory, i.e., when it attempts to visit a site for the first time. Here there is no fixed confining boundary; the condition depends on the history of the walk. But since the region explored by the walker grows $\propto t^{1 / 2}$, it effectively creates its own parabola.

It was recently shown [18] that an unbiased random walk on the nonnegative integers, with the origin absorbing, exhibits a continuously variable exponent $\delta$ when subject to a mobile, partial reflector. The latter is initially one site to the right of the walker. Each time the walker steps onto the site 
occupied by the reflector, it is reflected one step to the left with probability $r$ (it remains at its new location with probability $1-r$ ); in either case, the reflector is pushed forward one site in this encounter. The survival exponent $\delta=(1+r) / 2$ in this process [18]. Since the reflector effectively records the span of the walk (i.e., the rightmost site yet visited), its interaction with the walker represents a memory. We shall refer to this model as the soft reflector, to highlight the fact that the refector obligingly moves forward, even if the walker is reflected back. Note that in this case $\delta$ never exceeds unity.

The analysis of the random walk with soft reflector was subsequently extended to compact directed percolation [19]. The active region initially consists of a single site (the origin), and, as already noted, is bounded by a pair of independent, unbiased random walkers, originally at $x=0$ and $x=1$. The two walkers are subject to movable partial ("soft") reflectors, such that the walker on the right is reflected toward the left and vice-versa. The results for the survival exponent are qualitatively similar to those for the single walker, but $\delta$ now varies between $1 / 2$ and 1.160 as the reflection probability $r$ varies between zero and one. The results (coming in this case from iteration of the transition matrix, rather than from an asymptotic analysis of the generating function), are well fit by the simple expression $\delta=1 / 2+2 r / 3$; small but significant deviations from this simple formula are found, however. CDP with reflectors has so far defied exact analysis, and the reason for the specific value $\delta=1.160$ for $r=1$ is not understood.

Most recently, the methods developed in Ref. [18] were applied to a one-dimensional random walk with memory of a different form: if the target site $x$ lies in the region that has been visited before (that is, if $x$ itself has been visited, or lies between two sites that have been visited), then the step length is $v$; otherwise the step length is $n$. In this case one finds $\delta=v / 2 n$ [20]. Thus $\delta$ can take any rational value between zero and infinity.

With this background we may now describe the problem to be analyzed here as a random walk subject to a hard partial, movable reflector. This is because the reflector now moves forward if and only if the walker succeeds in occupying the new position; when the walker is reflected, the reflector maintains its position. This, as will be shown, can lead to much larger values of $\delta$ than in the soft reflector case.

Before embarking on the technical discussion, we summarize our approach, as developed in Refs. [18] and [20]. After formulating the problem, we enlarge the state space so that the process becomes Markovian in the expanded representation [21]. We then write down the equation of motion for the probability distribution and its associated boundary and initial conditions. Since these are discrete models, the equation of motion corresponds to a set of difference equations, first-order in time, and second-order in space. It is convenient to eliminate the time variable by passing to a generating function $\hat{P}(z)$ (effectively, a discrete Laplace transform). Using separation of variables, we obtain a formal solution for the generating function. Finally, the asymptotic long-time behavior is found by studying the generating function for the survival probability in the limit $z \rightarrow 1$.

\section{Model}

Consider an unbiased, discrete-time random walk on the nonnegative integers, with the origin absorbing. We denote the position of the walker at time $t$ by $x_{t}$, with $x_{0}=1$. The movement of the walker is affected by the presence of a movable partial reflector, whose position is denoted by $R_{t}$, with $R_{0}=2$. At each time step the walker hops from its current position $x_{t}$ to either $x_{t}+1$ or $x_{t}-1$ with probabilities of $1 / 2$. If, however, $x_{t}+1=R_{t}$, the walker is reflected back to $x_{t}$ with probability $r$, and remains at $x_{t}+1$ with probability $\bar{r} \equiv 1-r$; in the latter case the reflector simultaneously moves to $R_{t}+1$. Summarizing, the transition probabilities for the walker are

$$
x_{t+1}= \begin{cases}x_{t}-1 & , \text { w.p. } 1 / 2 \\ x_{t}+1 & , \text { w.p. } 1 / 2\end{cases}
$$

in case $x_{t} \leq R_{t}-2$. When $x_{t}=R_{t}-1$, we have instead

$$
x_{t+1}= \begin{cases}x_{t}-1 & , \text { w.p. } 1 / 2 \\ x_{t}+1 & , \text { w.p. } \bar{r} / 2 \\ x_{t} & , \text { w.p. } r / 2\end{cases}
$$

The position of the reflector at any moment is given by $R_{t}=1+\max _{t^{\prime} \leq t}\left\{x_{t^{\prime}}\right\}$.

Although the process $x_{t}$ is non-Markovian (since the transition probability into a given site depends on whether it has been visited previously), we can define a Markov process by expanding the state space to include the variable $y_{t} \equiv R_{t}-1=\max _{t^{\prime} \leq t}\left\{x_{t^{\prime}}\right\}$. The state space $E \subset \mathbb{Z}^{2}$ is given by by

$$
E=\left\{(x, y) \in \mathbb{Z}^{2}: x \geq 0, y \geq 1, \quad x \leq y\right\},
$$

as represented in Fig. 1.

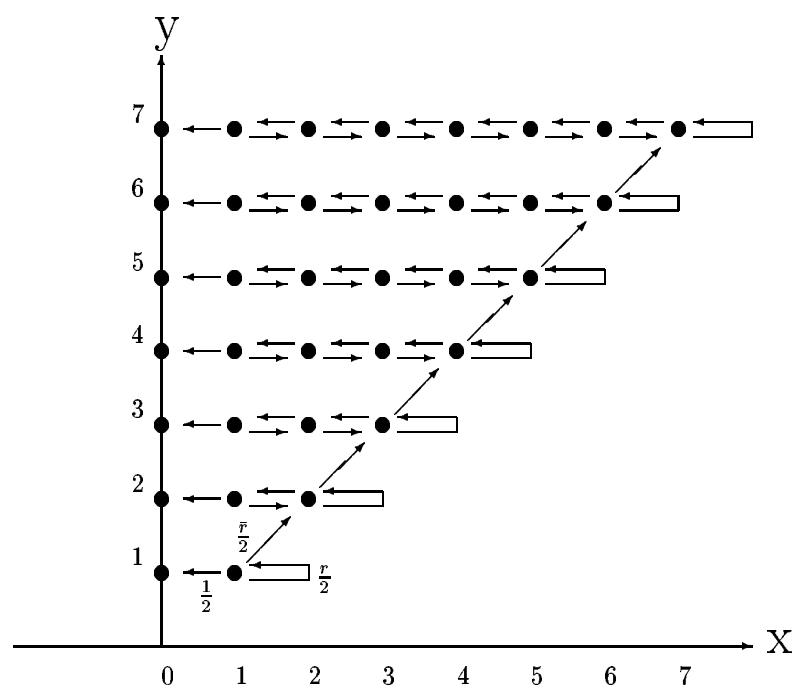

Figure 1. Random walk subject to hard reflector: transitions in the $x-y$ plane.

The probability distribution $P(x, y, t)$ follows the evolution equation 


$$
P(x, y, t+1)=\frac{1}{2} P(x+1, y, t)+\frac{1}{2} P(x-1, y, t), \quad \text { for } x<y-1,
$$

with $P(x, y, 0)=\delta_{x, 1} \delta_{y, 1}$. Eq. (3) is subject to two boundary conditions. The first is the absorbing condition for $x \leq 0$

$$
P(x, y, t)=0, \quad \text { for } x \leq 0 .
$$

The second applies along the diagonal $x=y$. Defining $D(y, t) \equiv P(y, y, t)$, we have

$$
D(y, t+1)=\frac{1}{2} P(y-1, y, t)+\frac{\bar{r}}{2} D(y-1, t)+\frac{r}{2} D(y, t), \quad \text { for } y \geq 2 .
$$

For $y=1$ the equation is simply $D(1, t+1)=(r / 2) D(1, t)$, and since $D(1,0)=1$, one has $D(1, t)=(r / 2)^{t}$. Finally, for $x=y-1$,

$$
P(y-1, y, t+1)=\frac{1}{2} P(y-2, y, t)+\frac{1}{2} D(y, t), \quad \text { for } x<y-1,
$$

We next introduce the generating function:

$$
\hat{P}(x, y, z)=\sum_{t=0}^{\infty} P(x, y, t) z^{t} .
$$

Multiplying Eqs. (3), (6) by $z^{t}$, summing over $t$ and shifting the sum index where necessary, one finds that the generating function satisfies

$$
\begin{aligned}
\frac{1}{z} \hat{P}(x, y) & =\frac{1}{2} \hat{P}(x+1, y)+\frac{1}{2} \hat{P}(x-1, y), & & \text { for } x \leq y-2 \\
\frac{1}{z} \hat{P}(y-1, y) & =\frac{1}{2} \hat{D}(y)+\frac{1}{2} \hat{P}(y-2, y), & & \text { for } x=y-1,
\end{aligned}
$$

(we drop the argument $z$ for brevity), where $\hat{D}(y)$ is defined by an expression analogous to Eq. (7). The initial condition implies $\hat{D}(1)=(1-z r / 2)^{-1}$; the boundary conditions are

$$
\hat{P}(0, y)=0
$$

and

$$
\frac{1}{z} \hat{D}(y)=\frac{1}{2} \hat{P}(y-1, y)+\frac{\bar{r}}{2} \hat{D}(y-1)+\frac{r}{2} \hat{D}(y), \quad \text { for } y \geq 2 .
$$

Eq. (8) relates $\hat{P}$ at different values of $x$, for the same $y$. Specifically, on the interior of each line of constant $y, \hat{P}$ satisfies a diffusion equation, with a source at $x=y$ and a sink at $x=0$. It is therefore natural to attempt separation of variables,

$$
\hat{P}(x, y)=\hat{A}(x) \hat{B}(y)
$$

Inserting this expression in Eq. (8) one obtains

$$
\frac{1}{z} \hat{A}(x)-\frac{1}{2} \hat{A}(x-1)-\frac{1}{2} \hat{A}(x+1)=0
$$

with $A(0)=0$. The solution satisfying this boundary condition is

$$
\hat{A}(x)=\lambda^{x}-\lambda^{-x}
$$

with

$$
\lambda=\frac{1}{z}+\sqrt{\frac{1}{z^{2}}-1} .
$$

Our next task is to determine $\hat{B}(y)$; for this we require a relation between generating functions with different arguments $y$. Relations of this kind arise along the diagonal, but involve the function $\hat{D}(y)$, which we proceed to eliminate. Combining Eqs. (9) and (11), we find

$$
Q(z) \hat{D}(y)=\frac{z \bar{r}}{2} \hat{D}(y-1)+\frac{z^{2}}{4} \hat{P}(y-2, y)
$$

where

$$
Q(z)=1-\frac{z r}{2}-\frac{z^{2}}{4}
$$


Equation (11) may also be written as

$$
\left(1-\frac{z r}{2}\right) \hat{D}(y)-\frac{z \bar{r}}{2} \hat{D}(y-1)=\frac{z}{2} \hat{P}(y-1, y)
$$

We now multiply Eq. (16) for $y-1$ by $\beta \equiv z \bar{r} /(2-z r)$ and subtract the result from the same equation for $y$. This yields

$$
Q[\hat{D}(y)-\beta \hat{D}(y-1)]=\frac{z \bar{r}}{2}[\hat{D}(y-1)-\beta \hat{D}(y-2)]+\frac{z^{2}}{4}[\hat{P}(y-2, y)-\beta \hat{P}(y-3, y-1)] .
$$

From Eq. (18) we have

$$
\hat{D}(y)-\beta \hat{D}(y-1)=\frac{z}{2-z r} \hat{P}(y-1, y),
$$

allowing us to eliminate $\hat{D}$ from Eq. (19):

$$
\frac{4 Q}{z(2-z r)} \hat{P}(y-1, y)-\hat{P}(y-2, y)=\beta\left[\frac{2}{z} \hat{P}(y-2, y-1)-\hat{P}(y-3, y-1)\right] \text {. }
$$

Inserting Eq. (12) one readily finds a recursion relation for $\hat{B}$ :

$$
\frac{\hat{B}(y)}{\hat{B}(y-1)}=\frac{z \bar{r}[2 \hat{A}(y-2)-z \hat{A}(y-3)]}{\left(4-2 z r-z^{2}\right) \hat{A}(y-1)-z(2-z r) \hat{A}(y-2)} .
$$

Given $\hat{B}(1)=\hat{D}(1) / \hat{A}(1)$ with $\hat{D}(1)$ and $\hat{A}(y)$ as found above, Eqs. (12), (14), and (22) represent a complete formal solution for the generating function $\hat{P}(x, y)$.

\section{Asymptotic analysis}

Our goal is to find the survival probability $S(t)$ for large $t$. This can be found analysing the associated generating function,

$$
\hat{S}(z)=\sum_{t=0}^{\infty} S(t) z^{t}
$$

in the limit $z \rightarrow 1$. Specifically, if $S(t) \sim t^{-\delta}$, then the radius of convergence of $\hat{S}(z)$ is $|z|=1$, and the singular behavior of the generating function as $z \rightarrow 1$ determines $\delta$. Indeed, in this case, with $z=1-\epsilon$, we have

$$
\begin{aligned}
\hat{S} & \simeq \sum_{t=1}^{\infty} t^{-\delta}(1-\epsilon)^{t} \\
& \simeq \int_{1}^{\infty} d t t^{-\delta} \exp [-t|\ln (1-\epsilon)|] \\
& \simeq \epsilon^{\delta-1} \Gamma(1-\delta),
\end{aligned}
$$

so that the scaling exponent $\delta$ can be read off from the power-law dependence of $\hat{S}$ on $\epsilon=1-z$ as $\epsilon \rightarrow 0$. This simplifies considerably the determination of the long-time asymptotic behavior of $S(t)$.

The generating function $\hat{S}$ has two contributions, coming from the "interior" $(x<y)$ and the diagonal:

$$
\begin{aligned}
\hat{S} & =\sum_{y=1}^{\infty} \sum_{x=1}^{y-1} \hat{P}(x, y)+\sum_{y=1}^{\infty} \hat{D}(y) \\
& \equiv \hat{S}_{P}+\hat{S}_{D} .
\end{aligned}
$$

Using Eq. (20) one readily shows that

$$
\hat{S}_{D}=\frac{z}{2-z} \sum_{y=1}^{\infty} \hat{P}(y-1, y)<\hat{S}_{P}
$$

so that it suffices to analyze the behavior of $\hat{S}_{P}$.

Consider

$$
\hat{S}_{P}=\sum_{y=1}^{\infty} \hat{B}(y) \sum_{x=1}^{y-1} \hat{A}(x)
$$

The sum over $x$ can be evaluated as

$$
\begin{aligned}
\sum_{x=1}^{y-1} \hat{A}(x) & =\frac{\lambda^{y}-1}{\lambda-1}-\frac{\lambda^{-y}-1}{\lambda^{-1}-1} \\
& \simeq \frac{4}{\Lambda} \sinh ^{2} \frac{\Lambda y}{2}
\end{aligned}
$$

where in the last step we used $\Lambda \equiv \ln \lambda \simeq \sqrt{2 \epsilon}$ as $\epsilon \rightarrow 0$.

To evaluate $\hat{S}_{P}$ we also require an expression for $\hat{B}(y)$ in the limit $\epsilon \rightarrow 0$; this can be obtained from Eq. (22). We begin by setting all explicit factors of $z$ equal to unity, since the $\mathcal{O}(\epsilon)$ corrections thereby discarded do not contribute to the singular behavior of $\hat{S}_{P}$. The singular contributions in fact originate from the functions $\hat{A}$, through their dependence on $\lambda$. Writing $\hat{A}(y)=2 \sinh \Lambda y$, we therefore have 


$$
\frac{\hat{B}(y)}{\hat{B}(y-1)} \simeq \frac{\bar{r}[2 \sinh \Lambda(y-2)-\sinh \Lambda(y-3)]}{(3-2 r) \sinh \Lambda(y-1)-(2-r) \sinh \Lambda(y-2)} .
$$

Using the identity $\sinh (a+b)=\sinh a \cosh b+$ $\sinh b \cosh a$, and neglecting terms $\mathcal{O}\left(\Lambda^{2}\right)$, we obtain

$$
\frac{\hat{B}(y)}{\hat{B}(y-1)} \simeq \frac{\tanh \Lambda y-\Lambda}{\tanh \Lambda y+\Lambda / \bar{r}}
$$

For $y \leq y_{0}=[2 / \bar{r}]+1$ (here $[\ldots]$ denotes the integer part of its argument), we can write, for small $\Lambda$

$$
\hat{B}(y)=\hat{B}(1) \prod_{k=2}^{y} \frac{k-1}{k+1 / \bar{r}}=C \hat{B}(1)
$$

where $C$ depends on $r$ and $y$ but is independent of $\Lambda$. We shall in fact discard the contribution due to $y<y_{0}$ in $\hat{S}_{P}$. The reason is that the contribution to the survival probability from any fixed, finite set of transient states must decay exponentially at long times, and so will not affect our result for the scaling exponent.

Noting that $\hat{B}(1)=\hat{D}(1) / \hat{A}(1) \propto 1 / \Lambda$, we have, for $y>y_{0}$,

$$
\hat{B}(y)=\frac{C}{\Lambda} \prod_{k=y_{0}}^{y} \frac{\hat{B}(k)}{\hat{B}(k-1)}
$$

where $C$ is a constant. Since all terms have $k \geq 2 / \bar{r}$, we may use Eq. (30) to write, with $\phi_{k} \equiv \tanh \Lambda k$,

$$
\begin{aligned}
\ln \frac{\hat{B}(y)}{\hat{B}\left(y_{0}\right)} & \simeq \sum_{k=y_{0}}^{y} \ln \frac{1-\Lambda / \phi_{k}}{1+\Lambda /\left(\bar{r} \phi_{k}\right)} \\
& \simeq-\left(1+\frac{1}{\bar{r}}\right) \Lambda \sum_{k=y_{0}}^{y} \frac{1}{\phi_{k}} .
\end{aligned}
$$

Approximating the sum by an integral we find

$$
\ln \frac{\hat{B}(y)}{\hat{B}\left(y_{0}\right)} \simeq\left(1+\frac{1}{\bar{r}}\right) \ln \frac{\sinh \Lambda y_{0}}{\sinh \Lambda y}
$$

Now, inserting Eqs. (28) and (34) in Eq. (27), the generating function for $\epsilon \rightarrow 0$ is:

$$
\hat{S}_{P} \sim \Lambda^{1 / \bar{r}-1} \sum_{y=y_{0}}^{\infty} \frac{\sinh ^{2} \Lambda y / 2}{\sinh ^{1+1 / \bar{r}} \Lambda y}
$$

where ' $\sim$ ' denotes asymptotic proportionality as $\epsilon \rightarrow 0$. Approximating, as before, the sum by an integral, we have

$$
\hat{S}_{P} \sim \Lambda^{1 / \bar{r}-2} \int_{\Lambda y_{0}}^{\infty} d u \frac{\sinh ^{2} u / 2}{\sinh ^{1+1 / \bar{r}} u}
$$

Since $1+1 / \bar{r} \geq 2$, the integral converges at its upper limit. For $r<1 / 2,1+1 / \bar{r}<3$ and the integral remains finite as $\Lambda \rightarrow 0$. Then

$$
\hat{S}_{P} \sim \Lambda^{1 / \bar{r}-2} \sim \epsilon^{1 /(2 \bar{r})-1}
$$

so that the survival probability decays as $S(t) \sim t^{-\delta}$ with $\delta=1 / 2 \bar{r}$. For $r=1 / 2$, the prefactor in Eq. (36) is independent of $\Lambda$ and

$$
\hat{S}_{P} \sim \int_{\Lambda y_{0}}^{\infty} \frac{d u}{u} \sim-\ln (1-z) .
$$

Expanding the logarithm, we find $\hat{S}(z) \sim \sum_{n} z^{n} / n$, yielding directly $S(t) \sim t^{-1}$. Finally, when $r>1 / 2,1+1 / \bar{r}>3$ and the integral in Eq. (36) contains two principal contributions: one finite (due to the interval from say, 1, to infinity), the other arising from the lower limit, and diverging as $\Lambda^{2-1 / \bar{r}}$. Combined with the prefactor $\propto \Lambda^{1 / \bar{r}-2}$ however, the latter contribution is nonsingular, while the former is again proportional to $\Lambda^{1 / \bar{r}-2}$.

Summarizing, the asymptotic survival probability decays as a power law,

$$
S(t) \sim t^{-1 / 2 \bar{r}}
$$

which is the result we set out to prove.

\section{Numerical Results}

The foregoing analysis provides the $t \rightarrow \infty$ scaling behavior of the survival probability, but does not indicate the rate of convergence to the asymptotic power law. To determine how the corrections to scaling decay, we iterate the discrete time evolution equation for $P(x, y, t)$ directly. In Fig. 2 we show the decay of $S(t)$ for reflection probability $r=0.85$, corresponding to $\delta=10 / 3$. For very late times, the graph indeed approaches a power law with the expected exponent. The approach is, however, extremely slow.

Since our asymptotic analysis only retains the leading dominant term in the long-time behavior of $S(t)$, we have no specific information on correction to scaling terms. It is easy to see, nonetheless, that corrections $\propto t^{-1 / 2}$ will be generated, since $\Lambda \simeq \sqrt{2 \epsilon}+\mathcal{O}(\epsilon)$. In fact, we are able to fit the long-time evolution of the survival probability by adding a suitable $\mathcal{O}\left(t^{-1 / 2}\right)$ term to the power law, but further terms $\left(\sim t^{-1}\right)$, etc. $)$ are required for intermediate times. 


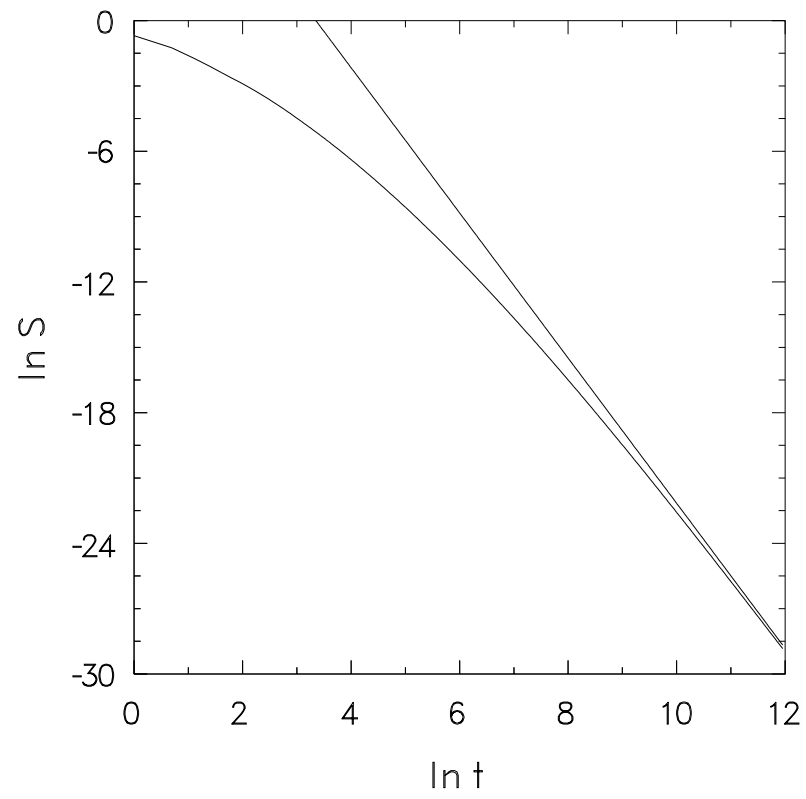

Figure 2. Decay of survival probability $S(t)$ for reflection probability $r=0.85$ (solid curve); the slope of the straight line is $-10 / 3$.

We have found a particularly simple transformation of variable that appears to take the dominant correction to scaling into account. It consists in defining a shifted time variable

$$
T=t+b t^{1 / 2}
$$

with parameter $b$ adjusted to make the graph of $S$ versus $T$ (on log scales) as linear as possible. Fig. 3 shows the survival probability data for $r=1 / 2,2 / 3,0.75$ and 0.85 versus $T$ (the corresponding $b$ values are $1.754,4.167,7.042$, and 16.67). In each case the numerical data (points) follow the modified power law,

$$
S(T)=A T^{-\delta},
$$

to very high precision. [Here $A$ is an amplitude determined by extrapolating $T^{\delta} S(T)$ to $T \rightarrow \infty$.] The numerical data appear to converge rapidly (faster than a power law) to the fit. While this 'shifted time' analysis is for the moment without theoretical basis, it clearly confirms the asymptotic power laws found analytically, and suggests a simple form for describing slowly decaying corrections to scaling.

The slowly decaying correction to scaling would likely frustrate efforts to extract the correct long-time behavior from simulations. Looking at Fig. 2, we see that the asymptotic power law is barely evident when $S(t)$ has decayed to $e^{-15}$. To obtain even marginally useful simulation data in this situation we would need to perform $\geq 10 e^{15} \simeq 3 \times 10^{7}$ independent realizations of the process, extending to a maximum time of about 2000 steps. This is feasible for a simple random walk, but becomes a computational challenge for a many-particle system. Thus, if lattice models such as the PCP behave in a manner analogous to what is found for the random walk with a hard reflector, it will be very difficult to confirm power-law scaling in simulations. Data for limited times (or limited samples) may well give the impression of faster than exponential decay of $S(t)$.

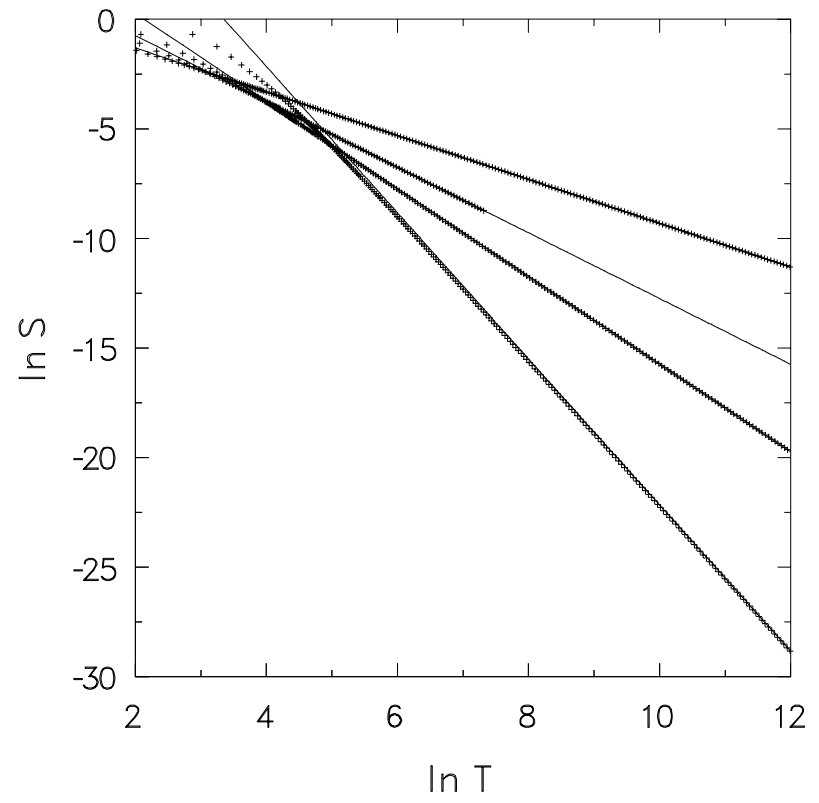

Figure 3. Survival probability $S$ as a function of the shifted time variable $T$, for reflection probabilities $r=1 / 2,2 / 3,3 / 4$ and 0.85 (data points); the straight lines have slopes of $-1,-3 / 2,-2$, and $10 / 3$.

\section{Discussion}

We have reviewed examples of confined random walks, and random walks with memory, that lead to a continuously variable scaling exponent for the survival probability, and analysed in detail the 'hard reflector' case. The latter problem appears to be particularly relevant to spreading in the pair contact process, since modification of the background density of isolated particles can only occur when activity invades a previously inactive region [8]. The strong correction to scaling found numerically for the hard reflector is reminiscent of the slow convergence (interpreted as faster than power-law decay in Ref. [6]), found in spreading studies of the PCP. Stretched-exponential decay of the survival probability has also been found via scaling arguments for an epidemic model with immunization [7].

Several interesting issues remain open. First, the nature of correction to scaling terms needs to be investigated using a more complete asymptotic expansion of the generating function. Second, one would like to understand the exponent values for CDP (obtained numerically in Ref. [19]) on the basis of the generating function approach. Finally, extension of any of the models discussed here to two or more dimensions promises to be a difficult but potentially fascinating challenge.

\section{Acknowledgments}

We thank Deepak Dhar and Miguel A. Muñoz for helpful discussions. R.D. and F.F.A. acknowledge financial support from CNPq (Brazil); D. b-A. acknowledges support of NSF (USA) under grant PHY-0140094. 


\section{References}

[1] M. N. Barber and B. W. Ninham, Random and Restricted Walks, (Gordon and Breach, New York, 1970).

[2] G. H. Weiss, Aspects and Applications of the Random Walk, (North Holland, Amsterdam, 1994).

[3] D. ben-Avraham and S. Havlin, Diffusion and Reactions in Fractals and Disordered Systems, (Cambridge University Press, Cambridge, 2000).

[4] K. De'Bell and T. Lookman, Rev. Mod. Phys. 65, 87 (1993).

[5] J. Marro and R. Dickman, Nonequilibrium Phase Transitions in Lattice Models, (Cambridge University Press, Cambridge, 1999).

[6] P. Grassberger, H. Chaté, G. Rousseau, Phys. Rev. E 55, 2488 (1997).

[7] A. Jimenez-Dalmaroni and H. Hinrichsen, preprint, condmat/0304113

[8] I. Jensen, Phys. Rev. Lett. 70, 1465 (1993); I. Jensen and R. Dickman, Phys. Rev. E 48, 1710 (1993).

[9] M. A. Muñoz, G. Grinstein, and R. Dickman, J. Stat. Phys. 91, 541 (1998).

[10] M. A. Muñoz, G. Grinstein, R. Dickman, and R. Livi, Phys. Rev. Lett. 76, 451 (1996).
[11] T. Bohr, M. van Hecke, R. Mikkelsen, and M. Ipsen, Phys. Rev. Lett. 86, 5482 (2001).

[12] H. Hinrichsen, Adv. Phys. 49, 815 (2000).

[13] T. E. Harris, Ann. Prob. 2, 969 (1974).

[14] E. Domany and W. Kinzel, Phys. Rev. Lett. 53, 311 (1984).

[15] P. Krapivsky and S. Redner, Am. J. Phys. 64, 546 (1996).

[16] L. Turban, J. Phys. A 25, L127 (1992); C. Kaiser and L. Turban, ibid., 27, L579 (1994).

[17] G. Ódor and N. Menyhárd, Phys. Rev. E 61, 6404 (2000).

[18] R. Dickman and D. ben-Avraham, Phys. Rev. E 64, 020102 (2001).

[19] R. Dickman and D. ben-Avraham, J. Phys. A 35, 7983 (2002).

[20] R. Dickman, F. F. Araujo Jr., and D. ben-Avraham, Phys. Rev. E 66, 051102 (2002).

[21] N. G. van Kampen, Stochastic Processes in Physics and Chemistry, (North-Holland, Amsterdam, 1992).

[22] E. W. Montroll, and G. H. Weiss, J. Math. Phys. 6, 167 (1965).

[23] F. Spitzer, Principles of Random Walk, (Springer, New York, 1976). 